\title{
Chapter 50 \\ Report on Global Environment \\ Competitiveness of El Salvador
}

El Salvador is located in Central America. It covers 20,700 square kilometres and borders the Pacific Ocean on the south, and the countries of Guatemala to the west and Honduras to the north and east. It covers 20.7 thousand of square kilometers and has a population of 6.23 million. Its GDP reaches $\$ 23.05$ billion in 2011 . Through the index system and evaluation model, the environment competitiveness index of El Salvador ranks at 59 in 133 countries.

Fig. 50.1 Contribution of

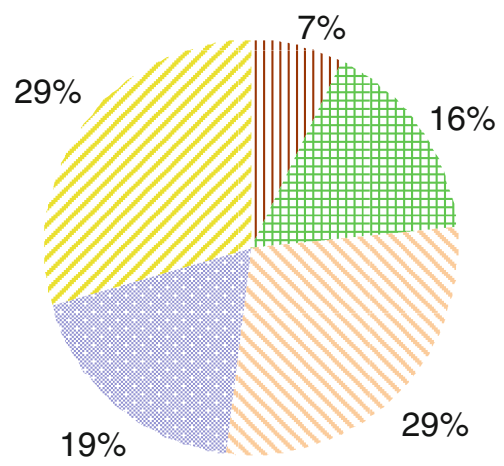

III Resource Environment Competitiveness

\# Ecological Environment Competitiveness Environment Carrying Competitiveness

* Environment Management Competitiveness Environment Harmony sub-index to GEC

Competitiveness 


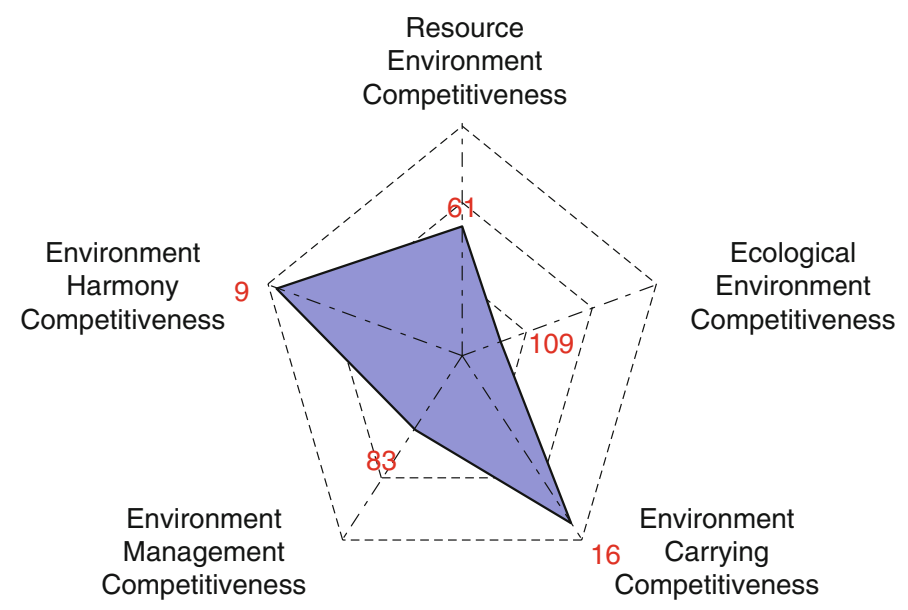

Fig. 50.2 Rank of sub-index of GEC

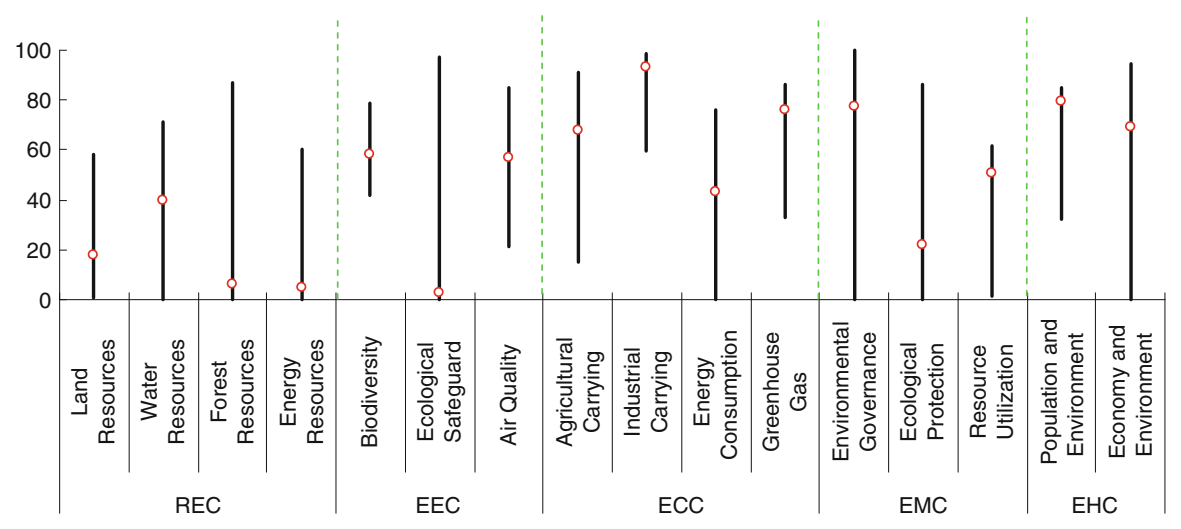

Fig. 50.3 Score and rank of the pillars of GEC

Table 50.1 Score and rank of all indicators of GEC

\begin{tabular}{|c|c|c|c|c|c|}
\hline Indicators & Score & Rank & Indicators & Score & Rank \\
\hline $\begin{array}{l}1 \text { Resource Environment } \\
\text { Competitiveness }\end{array}$ & 18.31 & 61 & $\begin{array}{l}\text { Total internal renewable } \\
\text { water resources }\end{array}$ & 43.20 & 26 \\
\hline 1.1 Land Resources & 18.05 & 37 & 1.3 Forest Resources & 6.48 & 126 \\
\hline Land area per capita & 0.58 & 120 & Growing stock in forest & N/A & N/A \\
\hline $\begin{array}{l}\text { Percentage of arable land to } \\
\text { total land area }\end{array}$ & 54.41 & 19 & $\begin{array}{l}\text { and other wooded land } \\
\text { Proportion of land area }\end{array}$ & 15.97 & 85 \\
\hline Arable land per capita & 4.99 & 95 & covered by forest & & \\
\hline 1.2 Water Resources & 39.59 & 22 & Forest area per capita & 0.32 & 110 \\
\hline Surface water & 13.06 & 23 & 1.4 Energy Resources & 5.08 & 68 \\
\hline Annual precipitation & 59.35 & 22 & Fossil energy & 0.00 & 64 \\
\hline Groundwater & 42.73 & 14 & Energy production & 0.37 & 99 \\
\hline
\end{tabular}


Table 50.1 (continued)

\begin{tabular}{|c|c|c|c|c|c|}
\hline \multirow{3}{*}{$\begin{array}{l}\text { Indicators } \\
\text { Proportion of combustible } \\
\text { renewable and waste to } \\
\text { total energy consumption }\end{array}$} & Score & Rank & Indicators & Score & Rank \\
\hline & 19.70 & 43 & $\begin{array}{l}\text { Energy consumption } \\
\text { per unit of land area }\end{array}$ & 99.57 & 90 \\
\hline & & & Ratio of clean energy & 48.14 & 10 \\
\hline $\begin{array}{l}\text { Net energy imports of the } \\
\text { energy consumption }\end{array}$ & 6.77 & 83 & $\begin{array}{l}\text { consumption } \\
\text { Elasticity of energy }\end{array}$ & 14.40 & 32 \\
\hline 2 Ecological Environment & 41.00 & 109 & consumption & & \\
\hline Competitiveness & & & Elasticity of electric power & 10.41 & 78 \\
\hline 2.1 Biodiversity & 58.22 & 62 & consumption & & \\
\hline Threatened fish species & 93.40 & 41 & 3.4 Greenhouse Gas & 76.09 & 8 \\
\hline $\begin{array}{l}\text { Threatened mammal } \\
\text { species }\end{array}$ & 97.28 & 22 & $\begin{array}{l}\text { Growth rate of } \mathrm{CO}_{2} \\
\text { emissions }\end{array}$ & 74.59 & 11 \\
\hline Threatened plant species & 98.60 & 80 & Growth rate of Methane & 67.32 & 17 \\
\hline GEF benefits index for & 0.90 & 84 & emissions & 0060 & 80 \\
\hline 2.2 Ecological Safeguard & 2.73 & 120 & of land area & & \\
\hline Terrestrial protected areas & 1.90 & 121 & $\mathrm{CO}_{2}$ emissions per unit of & 64.24 & 29 \\
\hline Marine protected areas & 3.98 & 49 & energy consumption & & \\
\hline 2.3 Air Quality & 56.80 & 72 & 4 Environment Management & 47.35 & 83 \\
\hline Inhalable particles (PM10) & 79.56 & 66 & Competitiveness & & \\
\hline Particulate matter (PM2.5) & 89.35 & 37 & 4.1 Environmental & 77.67 & 90 \\
\hline $\begin{array}{l}\text { Index of indoor air } \\
\text { pollution }\end{array}$ & 21.90 & 76 & $\begin{array}{l}\text { Governance } \\
\text { Agricultural chemicals }\end{array}$ & 66.67 & 65 \\
\hline Nitrogen oxides emission & 68.72 & 29 & regulation & & \\
\hline Sulfur dioxide emission & 40.92 & 25 & Percentage of the rural & 76.00 & 84 \\
\hline $\begin{array}{l}3 \text { Environment Carrying } \\
\text { Competitiveness }\end{array}$ & 72.25 & 16 & $\begin{array}{l}\text { population with access to } \\
\text { an improved water } \\
\text { source }\end{array}$ & & \\
\hline 3.1 Agricultural Carrying & 67.86 & 57 & Percentage of the urban & 94.00 & 97 \\
\hline $\begin{array}{l}\text { Cereal yield per unit of } \\
\text { arable land }\end{array}$ & 27.47 & 68 & $\begin{array}{l}\text { population with access to } \\
\text { an improved water }\end{array}$ & & \\
\hline $\begin{array}{l}\text { Fertilizer consumption per } \\
\text { unit of arable land }\end{array}$ & 91.32 & 82 & source & & \\
\hline $\begin{array}{l}\text { Annual freshwater } \\
\text { withdrawals for }\end{array}$ & 98.26 & 71 & $\begin{array}{l}\text { 4.2 Ecological Protection } \\
\text { Area of plantation and } \\
\text { afforestation }\end{array}$ & $\begin{array}{r}22.10 \\
0.02\end{array}$ & $\begin{array}{l}100 \\
108\end{array}$ \\
\hline $\begin{array}{l}\text { agriculture per unit of } \\
\text { arable land }\end{array}$ & & & Biome protect & 4.90 & 122 \\
\hline 3.2 Industrial Carrying & 93.48 & 43 & Overfishing of fishing & 68.75 & 20 \\
\hline $\begin{array}{l}\text { Net exports of goods as a } \\
\text { percentage of GDP }\end{array}$ & 85.45 & 41 & 4.3 Resource Utilization & 50.69 & 13 \\
\hline Electric power consumption & 93.38 & 51 & $\begin{array}{l}\text { Utilization rate of water } \\
\text { resources }\end{array}$ & 0.22 & 73 \\
\hline of industry & & & Percentage of total internal & 56.84 & 80 \\
\hline $\begin{array}{l}\mathrm{SO}_{2} \text { emissions per unit of } \\
\text { value added of industry }\end{array}$ & 99.87 & 50 & $\begin{array}{l}\text { renewable water } \\
\text { resources to total water } \\
\text { resources }\end{array}$ & & \\
\hline $\begin{array}{l}\text { Annual freshwater } \\
\text { withdrawals for industry }\end{array}$ & 95.23 & 91 & $\begin{array}{l}\text { Percentage of agricultural } \\
\text { land to total land area }\end{array}$ & 88.08 & 11 \\
\hline & & & Percentage of fossil fuel & 57.64 & 27 \\
\hline 3.3 Energy Consumption & 43.13 & 17 & $\begin{array}{l}\text { energy consumption to } \\
\text { total energy consumption }\end{array}$ & & \\
\hline
\end{tabular}


Table 50.1 (continued)

\begin{tabular}{|c|c|c|c|c|c|}
\hline Indicators & Score & Rank & Indicators & Score & Rank \\
\hline 5 Environment Harmony & 74.31 & 9 & $\mathrm{CO}_{2}$ emissions per capita & 97.54 & 36 \\
\hline Competitiveness & & & Energy consumption per & 95.77 & 32 \\
\hline 5.1 Population and & 79.61 & 16 & capita & & \\
\hline Environment & & & 5.2 Economy and & 69.01 & 38 \\
\hline Percentage of population & 87.00 & 67 & Environment & & \\
\hline $\begin{array}{l}\text { with access to Improved } \\
\text { sanitation facilities }\end{array}$ & & & $\begin{array}{l}\text { Land resource utilization } \\
\text { efficiency }\end{array}$ & 0.32 & 36 \\
\hline $\begin{array}{l}\text { Motor vehicles per } 1,000 \\
\text { people }\end{array}$ & 89.88 & 50 & $\begin{array}{l}\text { Sulfur dioxide emissions per } \\
\text { unit of GDP }\end{array}$ & 95.95 & 56 \\
\hline $\begin{array}{l}\text { Renewable internal } \\
\text { freshwater resources per }\end{array}$ & 3.45 & 68 & $\begin{array}{l}\text { Carbon dioxide emissions } \\
\text { per unit of GDP }\end{array}$ & 91.66 & 45 \\
\hline $\begin{array}{l}\text { capita } \\
\mathrm{SO}_{2} \text { emissions per capita }\end{array}$ & 98.13 & 38 & $\begin{array}{l}\text { Energy consumption per unit } \\
\text { of GDP }\end{array}$ & 88.09 & 50 \\
\hline
\end{tabular}

Table 50.2 Rank distribution of the individual indicators of GEC

\begin{tabular}{|c|c|c|c|c|c|c|}
\hline Sub-index & $\begin{array}{l}\text { Number } \\
\text { of the } \\
\text { individual } \\
\text { indicators }\end{array}$ & $\begin{array}{l}\text { Rank } \\
1-10\end{array}$ & $\begin{array}{l}\text { Rank } \\
11-30 \\
\end{array}$ & $\begin{array}{l}\text { Rank } \\
31-60 \\
\end{array}$ & $\begin{array}{l}\text { Rank } \\
61-100 \\
\end{array}$ & $\begin{array}{l}\text { Rank } \\
101-133\end{array}$ \\
\hline $\begin{array}{l}\text { Resource Environment } \\
\text { Competitiveness }\end{array}$ & 14 & 0 & 5 & 2 & 4 & 2 \\
\hline $\begin{array}{l}\text { Ecological Environment } \\
\text { Competitiveness }\end{array}$ & 11 & 0 & 2 & 2 & 5 & 2 \\
\hline $\begin{array}{l}\text { Environment Carrying } \\
\text { Competitiveness }\end{array}$ & 15 & 2 & 3 & 6 & 4 & 0 \\
\hline $\begin{array}{l}\text { Environment Management } \\
\text { Competitiveness }\end{array}$ & 10 & 0 & 2 & 0 & 6 & 2 \\
\hline $\begin{array}{l}\text { Environment Harmony } \\
\text { Competitiveness }\end{array}$ & 10 & 0 & 1 & 7 & 2 & 0 \\
\hline Total & 60 & 2 & 13 & 17 & 21 & 6 \\
\hline
\end{tabular}

Open Access This chapter is distributed under the terms of the Creative Commons Attribution Noncommercial License, which permits any noncommercial use, distribution, and reproduction in any medium, provided the original author(s) and source are credited. 